\section{Investigar sobre extensión. El caso de la Universidad Nacional de Entre Ríos}

Andrés Gabriel Wursten

Consejo Nacional de Investigaciones

Científicas y Técnicas. Universidad Nacional

de Entre Ríos, Argentina.

wurstenandres@gmail.com
Investigación y extensión universitaria /

Perspectivas
RECEPCIÓN: 29/05/18

ACEPTACIÓN FINAL: 28/06/18

\section{Resumen}

El presente año supone un punto de inflexión para la extensión universitaria al cumplirse el centenario de la Reforma de 1918. En este sentido, los análisis sobre su devenir histórico y actualidad coinciden en señalar una serie de desafíos pendientes para el campo: 1) incorporarla en el currículo de las carreras; 2) valorarla como un trabajo académico en paridad de condiciones con la docencia y la investigación; y 3) fortalecer la integración de las funciones universitarias. En este artículo se propone añadir un cuarto aspecto a esa agenda: incrementar el desarrollo de investigaciones cuyo objeto de estudio sea la extensión con el propósito de generar un cuerpo de conocimiento en ese ámbito que acompañe a los objetivos anteriores y fortalezca dicha función. En esta dirección, se presenta una propuesta de esa índole anclada en la Universidad Nacional de Entre Ríos, sus principales características y resultados preliminares.

Palabras clave: extensión, investigación universidad, apropiación social del conocimiento científico
Investigate about extension. The case of the National University of Entre Ríos

\section{Abstract}

This year constitutes a turning point for university extension at the centennial of the 1918 Reform. In this sense, the analyzes of its historical evolution and current events coincide in pointing out a series of pending challenges for the field: 1) incorporate it into the racing curriculum; 2) evaluate it as an academic work in parity of conditions with teaching and research; 3) strengthen the integration of university functions. This article proposes to add a fourth aspect to this agenda: to increase the development of research whose object of study is extension, with the purpose of generating a body of knowledge in the field that accompanies the previous objectives and strengthens this function. In this direction, a proposal of that nature anchored in the National University of Entre Ríos, its main characteristics and preliminary results is presented.

Keywords: extension, research, university, social appropriation of scientific knowledge
Investigue sobre extensão. O caso da Universidade Nacional de Entre Rios

\section{Resumo}

Este ano marca um ponto de virada para a extensão universitária no centenário da Reforma de 1918. Nesse sentido, as análises sobre sua evolução histórica e eventos atuais coincidem em apontar uma série de desafios pendentes para o campo: 1) incorporá-la no currículo das carreiras universitárias; 2) valorizá-la como um trabalho acadêmico em paridade de condições com ensino e pesquisa; 3) fortalecer a integração das funções universitárias. Este artigo propõe adicionar um quarto aspecto a esta agenda: aumentar o desenvolvimento de pesquisas cujo objeto de estudo é a extensão, com o objetivo de gerar um corpo de conhecimento no campo que acompanha os objetivos anteriores e fortalece essa função. Nessa direção, apresenta-se uma proposta dessa natureza ancorada na Universidad Nacional de Entre Ríos, suas principais características e resultados preliminares.

Palavras-chave: textensão, pesquisa, universidade, apropriação social do conhecimento científico

Para citación de este artículo: Wursten, A. (2018). Investigar sobre extensión. El caso de la Universidad Nacional de Entre Ríos. +E: Revista de Extensión Universitaria, 8(8), enero-junio, 26-43. doi: 10.14409/extensión.v8i8.Ene-Jun.7712. 


\section{Introducción}

El presente año supone un punto de inflexión para la universidad en general, y para la función de extensión en particular, al cumplirse el centenario de la Reforma de Córdoba de 1918. Esta se impuso contra el régimen conservador vigente, promovió un conjunto de cambios políticosociales tanto internos a la academia —autonomía, participación estudiantil en el gobierno, libertad de cátedra, fomento a la investigación - como los relacionados con la articulación entre universidad y sociedad -democratización de las instituciones, mayor acceso de la población y el compromiso de participar en los procesos culturales y productivos del país.

Durante la década de 1920, la experiencia se propagó por la región y representó un despertar de las universidades latinoamericanas. Son diversos los análisis que, tomando como antecedentes los movimientos reformistas, analizan el devenir histórico y caracterizan la extensión universitaria como campo que adquiere particularidades específicas en ese contexto (Arocena, s/f; Cano Menoni, 2014; Castro y Tommasino, 2017; Castro y Oyarbide, 2015; Cecchi, Pérez y Sanllorenti, 2013; Menéndez, 2017). Estos estudios coinciden, también, en proponer una serie de estrategias con vistas a fortalecerla en la actualidad. En primer lugar, abogan por su incorporación al currículo de las carreras y, entendiendo que la extensión adolece de reconocimiento respecto de la docencia y la investigación, se plantea colocar la actividad en simetría con estas; se trata, en otros términos, de fomentar su jerarquización. Asimismo, se reclama una integración más profunda entre las funciones clásicas que tienda a superar las barreras que fragmentan y compartimentalizan el accionar académico.

Las problemáticas planteadas en el ámbito teórico son, a su vez, acompañadas por las políticas universitarias. Como forma de aunar esfuerzos y desarrollar el campo de la extensión a nivel regional hay redes específicas en la temática —como la Unión Latinoamericana de Extensión Universitaria (ULEU) - y otras que han integrado la cuestión en sus comisiones de trabajo -es el caso de la Asociación Universitaria Grupo Montevideo (AUGM)—. Existen además experiencias destacables en cuanto a las estrategias de integralidad de las funciones, entre las cuales la Universidad de la República (UdelaR, Uruguay) es el referente principal. A partir de las propuestas de Espacios de Formación Integral y el Programa Integral Metropolitano, la UdelaR trabaja la extensión como práctica pedagógica fuertemente vinculada con procesos investigativos; de este modo, como sostiene Arocena (s/f), el tridente conforma un circuito en el que dependen la una de la otra. También en Argentina se vienen desarrollando distintas iniciativas orientadas en esta dirección. En 2008 se creó la Red Nacional de Extensión Universitaria, uno de cuyos propósitos es lograr la implementación de la extensión como actividad curricular (Estatuto REXUNI). Por su parte, la Universidad Nacional de Entre Ríos (UNER) ha incorporado líneas específicas en su Sistema de Proyectos de Extensión tendientes a resolver las problemáticas planteadas, tales como: Programas de Extensión, Proyectos de Curricularización, Proyectos de Integración de Funciones Universitarias, Proyectos de Extensión de Alumnos Avanzados. ${ }^{1}$

Como puede observarse, tanto desde las reflexiones con base en teorías y prácticas como desde las políticas universitarias, el campo de la extensión latinoamericana está empeñado en revalorizar la actividad e integrarla a las otras funciones. Sin embargo, es preciso señalar cierta carencia de investigaciones que aborden de manera específica la problemá- 
tica de la extensión y que acompañen las estrategias planteadas anteriormente. Solo por señalar un dato preliminar como sustento de esa valoración, de 32 artículos incluidos en el número anterior de esta revista, ${ }^{2}$ solamente cuatro (Beltramino y Theiler, 2017; Bolaños, Lara Campos y Villalobos Araya, 2017; González López, 2017; Lossio y Ruben, 2017) presentan argumentos producidos en el contexto de investigaciones sobre la temática. Por ello, la actual convocatoria de $+E$ significa un aporte sustantivo al respecto al establecer como tema central "Investigación y extensión universitaria. La misión social como campo de estudio". Se trata de fomentar investigaciones a partir de la cuales se comprenda a la extensión como campo problemático particular y de consolidar un cuerpo de conocimientos que contribuya a su integralidad, curricularización y jerarquización.

Antes de continuar, conviene formular algunas aclaraciones. Como se señaló en párrafos previos, existen numerosos aportes teóricos y prácticos sobre la temática extensionista cuyos logros más importantes han sido analizar en profundidad la singularidad de las experiencias latinoamericanas, marcar los derroteros históricos y las agendas actuales. Lejos se está de pretender marginalizar o restar valor al trayecto ya recorrido y los conocimientos acumulados, reemplazándolos por una visión "academicista". Lo que se propone, más bien, es que entre los desafíos propios del campo se enfatice la necesidad de producir saberes desde la investigación, de, sobre y desde las prácticas que, a su vez, contribuyan a retroalimentarlas. Por otra parte, podría objetarse que en el propósito de "integralidad" estaría presente, de distintas formas, la vinculación entre extensión e investigación. Por ejemplo, con referencia a proyectos con una comunidad determinada encarados desde ambas dimensiones; en actividades con colectivos sociales de las cuales surjan inquietudes que ulteriormente se traduzcan en investigaciones o bien, a la inversa, resultados de estudios empíricos que demanden una intervención. Pero esta clase de articulación no necesariamente hace referencia específica al problema que aquí nos ocupa: el de la extensión como objeto de estudio.

En este artículo se parte de la base de que la investigación sobre la extensión debe considerarse una de las principales estrategias que contribuyen (o deberían contribuir) a su fortalecimiento. Esa es la premisa que orienta la tesis doctoral "Apropiación Social del Conocimiento Científico. Análisis de la práctica extensionista en la Universidad Nacional de Entre Ríos", 3 cuyos lineamientos generales y avances se ponen en discusión en las páginas siguientes. En primer lugar, se describen brevemente los enfoques teóricos que sustentan el trabajo. A continuación, se presenta el caso de estudio -el sistema de programas, proyectos y acciones de extensión de la UNER-y se detallan los objetivos y metodología del estudio empírico en curso. En la sección "Resultados preliminares" se ponen en discusión los avances logrados hasta el momento producto del análisis documental y de entrevistas en profundidad con informantes clave. Para finalizar, se incluye una reflexión sobre las agendas actuales de la extensión y las posibles contribuciones que el enfoque propuesto representa para el campo.

2) Excluyendo las secciones de Reseña de Libros, Apuestas y Agenda Redes, que en su totalidad representan 8 artículos. 3) El trabajo en cuestión corresponde a una tesis presentada para el Doctorado en Ciencias Sociales de la UNER cuya culminación está prevista para 2019. Se desarrollan los principales aportes sobre la extensión y se dejan al margen los análisis centrados en la comunicación pública de la ciencia y comunicación comunitaria. 


\section{Extensión y apropiación social del conocimiento científico: convergencias conceptuales y desafíos}

Como se mencionó, este artículo se confecciona sobre la base de un trabajo de tesis doctoral que tiene como objeto de estudio la extensión en la UNER y cuyo marco teórico integra el propio campo de la extensión universitaria con las perspectivas de comunicación pública de la ciencia y comunicación comunitaria. Se parte de comprender que las prácticas orientadas a compartir el conocimiento científico y promover su apropiación social conforman actualmente un campo de interés teórico y práctico. La relevancia del tema se debe, como señala Cortassa, a su problemática multidimensional política, epistémica y cultural.

“(...) política, en primer lugar, porque se encuentran ligadas al ejercicio de los derechos y responsabilidades de los ciudadanos en un sistema democrático de participar en los debates sobre temas que les afectan; epistémica, porque los agentes expertos y no expertos se encuentran en desiguales condiciones en relación con el saber especializado; y cultural, porque el proceso se ve constreñido por una serie de representaciones simbólicas que inciden sobre su curso y resultados". (2012:15)

Es en ese sentido que el reclamo siempre presente por la democratización del conocimiento revitaliza su relevancia y se fortalece el interés por los procesos de apropiación social del conocimiento científico y técnico (en adelante, ASCCT) como acción propositiva de los ciudadano.

La universidad, como institución de producción y circulación de la ciencia, vincula principalmente, a partir de la extensión, los distintos sectores sociales, tanto la comunidad académica - estudiantes, docentes, administrativos - como aquellos que no forman parte directa de la misma -trabajadores, empresarios, colectivos específicos, ciudadanía en general一. Al respecto, puede pensarse la extensión como espacio simbólico de interfaz entre los sectores a partir del cual se generan procesos de producción y circulación de los conocimientos científico-técnicos.

En ese marco general, la propuesta es situar la problemática de la ASCCT en las prácticas extensionistas. De esta articulación emerge un conjunto de interrogantes valiosos para la investigación de la extensión, entre otros: ¿qué concepciones -implícitas o explícitas- de ASCCT subyacen a las prácticas de extensión? ¿De qué manera esas perspectivas se reflejan en mayor o menor medida - o no se reflejan en absoluto- en las estrategias y acciones desplegadas en el territorio? ¿Cómo evalúan esas prácticas los diferentes actores participantes en relación con el objetivo de promover formas eficaces de apropiación colectiva del conocimiento? ¿Cómo potenciar — teórica y metodológicamente— la circulación del conocimiento y la participación ciudadana en su elaboración y distribución a partir de las perspectivas de comunicación pública de las ciencias, de comunicación comunitaria y extensionistas?

Si bien el propósito de la mencionada tesis es abordar los procesos de apropiación social del conocimiento mediante una triangulación conceptual a priori novedosa, queda claro que los aportes de los estudios sobre extensión constituyen su eje vertebrador fundamental. Por esa razón, a continuación se sintetiza una serie de planteamientos conceptuales básicos atinentes como paso previo a describir en profundidad la investigación en curso, cuyos avances se exponen en páginas subsiguientes. 


\section{La extensión desde una mirada latinoamericana}

La primera etapa de la investigación aborda el devenir histórico de la extensión universitaria y sus particularidades en la región, a partir de lo cual se pueden inferir, entre los distintos análisis, puntos en común que configuran su campo actual.

En primer lugar, se observa un reconocimiento crítico de la multiplicidad de acepciones y valoraciones sobre lo que se denomina extensión, tanto a nivel discursivo como en las prácticas y actividades. Un dato significativo es la publicación del Glosario de términos que se utilizan en extensión universitaria (ULEU, 2015), donde se reconoce la diversidad conceptual y nominal del campo en América Latina. Esta constelación de conceptos no es considerada una condición negativa; al contrario, se reconoce una riqueza en la complejidad de las prácticas universitaria para lograr una labor transdisciplinaria y de integralidad de funciones.

En segundo lugar, en la diversidad de perspectivas a partir de las cuales se debate y desarrolla la extensión puede identificarse la existencia de, al menos, tres modelos (Valsagna, 2009; Tommasino y Cano, 2016). El primero, vinculado a la tradición iluminista en que se crearon las universidades modernas, confiado en los beneficios de la ciencia para las sociedades. En este caso, los expertos poseen un rol preponderante como los portadores del saber sobre otros actores que se entienden carentes de conocimiento relevante. Por otra parte, se puede observar -influenciada por el éxito del modelo alemán y norteamericanola extensión como el anclaje con el sector económico-productivo, que inmediatamente se homologa con el bienestar de toda la sociedad. Aquí impera el rol universitario de transferencia y/o venta de servicios, que supone la mejor opción para el desarrollo económico y social.

En tercer lugar, otra postura cuestiona las implicancias de los postulados de la ciencia moderna así como el extremo economicismo imperante en el modelo de las entrepreneurial universities (Pérez Lindo, 2003). Es aquella que retoma los aportes reformistas junto a trabajos de otros autores, como Frondizi y Freire, y reivindica una corriente de pensamiento latinoamericana en profundo conocimiento de las realidades regionales de desigualdad y pobreza generadas por el contexto de capitalismo y globalización. Reclama, entonces, un proceso de comunicación entre universidad y sociedad que reconozca las voces de los distintos actores en busca de la transformación colectiva de las comunidades.

A pesar de la diversidad encontrada en el campo, el análisis muestra que el discurso extensionista se basa en una arraigada fe en el conocimiento y en la universidad como su principal centro de producción. Valsagna (2009) explica que en todas las tradiciones el punto en común es considerar al conocimiento como poder transformador de la sociedad. Por su parte, Cecchi et al. (2013) emplea la misma hipótesis al plantear que el insumo clave de la transformación social es el conocimiento, razón por la cual debe gestionárselo a partir del compromiso social.

El conjunto de los aportes de los autores referenciados entiende la función de extensión, en términos generales, como el vínculo que la universidad mantiene con su entorno. En algunos casos, esta relación se basa en un modelo vertical de transferencia a partir de prácticas de sesgos paternalistas y asistencialistas; en otros, el énfasis está puesto en una noción productivo-mercantilista de venta de conocimientos; y finalmente, encontramos experiencias que trabajan sobre la idea de compromiso de la academia con la comunidad como acción política y ética de integración dialógica. 
Asimismo, existe coincidencia en la distinción de la extensión como uno de los pilares universitarios junto con la docencia y la investigación, mencionada como tercera función producto del devenir histórico de la universidad occidental y particularmente latinoamericana. Se señalan sus primeros antecedentes en la región a finales del siglo XIX y principios del XX, cuyos mayores referentes son la Reforma Universitaria de Córdoba y los procesos emancipatorios producidos en las universidades latinoamericanas inmediatamente después. Con posterioridad, los aportes reformistas se complementan en especial con la crítica freiriana, desde la cual se entiende la relación extensionista como un proceso dialógico en el que la comunidad universitaria y el pueblo - a partir de sus saberes particulares- construyen un conocimiento de carácter democrático, emancipatorio y transformador de la realidad (Cano Menoni, 2014; Cecchi et al., 2013; Pérez Lindo, 2003; Tünnermann Bernheim, 2003).

Dentro de sus balances, los autores enuncian una serie de deudas pendientes que exhortan a trabajar en la actualidad (Arocena, s/f; Cano Menoni, 2014; Castro y Tommasino, 2017; Castro y Oyarbide, 2015; Cecchi, et al., 2013). Apuntan al deber de fortalecer la extensión a partir de las siguientes estrategias:

1) Curricularización. La problemática de la extensión debe ser incorporada al currículo de las distintas carreras.

2) Jerarquización. Puesto que se entiende que la extensión carece de reconocimiento académico respecto de la docencia y la investigación, se plantea colocar la actividad en simetría con las demás funciones; y en este sentido, la pretensión principal es que se transforme en un antecedente de igual valor en los concursos académicos.

3) Integración. Se refiere a generar un trabajo sinérgico entre las funciones superando las barreras que dividen el accionar universitario.

Las actuales propuestas impulsan cambios epistémicos y pedagógicos en cuanto a los modelos tradicionales. En primera instancia, se incorporan los saberes populares en un intento por desmitificar la ciencia como valor imperante por encima de otras formas de conocimiento, colocándola como un modo particular de participación en una construcción colectiva. Esto se observa particularmente en el enfoque de la "ecología de saberes" propuesto por Sousa Santos (2007), el cual sostiene que la ciencia establece un diálogo con otros conocimientos sociales desprestigiados por el proceso de colonización cultural. Esta visión dialógica es eje central de la relación entre los actores participantes. Y lo anterior permite deducir que la docencia ya no puede ser entendida como la práctica en la cual el profesor transfiere el conocimiento hacia el estudiante ignorante -lo que Freire (1970) denominó "educación bancaria" - sino que la misma se funda en una simetría desprovista de roles estructurados, en un proceso donde los diferentes agentes intercambian conocimientos y experiencias sin que haya relaciones de jerarquía.

Finalmente, es tal la importancia del movimiento de 1918 que algunos autores proponen una "Segunda Reforma" que transforme la universidad latinoamericana otorgándole un rol central en el futuro de los países, el desarrollo cultural y económico de la región (Sousa Santos, 2007; Arocena, s/f; Castro y Oyarbide, 2015). El contexto actual está marcado por un sistema global capitalista que coloca a nuestra región en relaciones asimétricas y dependientes, lo que trae aparejados la desigualdad y el empobrecimiento al interior de las sociedades. 
Es menester de la universidad en general, y de las prácticas extensionistas en particular, ser partícipes de un proceso que revierta estas realidades, acercándose a las minorías y grupos más vulnerados en busca de la equidad social.

\section{Una propuesta de investigación empírica de la extensión en la UNER. Presentación del caso}

La UNER se creó en 1973 y pertenece al sistema público de educación superior de Argentina. Actualmente, cuenta con nueve unidades académicas distribuidas en distintas localidades de la provincia de Entre Ríos: Facultades de Ciencias Económicas, Ciencias de la Educación, Trabajo Social (Paraná); Facultades de Ciencias Agropecuarias y de Ingeniería (Oro Verde); Facultades de Ciencias de la Alimentación y Administración (Concordia); Facultad de Ciencias de la Salud (Concepción del Uruguay); subsede de la Facultad de Ciencias de la Salud (Villaguay); Facultad de Bromatología (Gualeguaychú).

A nivel político-normativo, la UNER está en consonancia con ciertas líneas desarrolladas en el apartado anterior respecto de la extensión latinoamericana. En su Estatuto (Resolución CS 113/05) se afirma que a la Universidad le corresponde desarrollar iniciativas que busquen difundir y transferir la ciencia, tecnología y cultura de acuerdo con las necesidades sociales contribuyendo al desarrollo nacional y regional. Estas acciones deben estudiar los problemas del medio con la articulación de los diferentes estamentos de la academia y la comunidad.

En ese marco general, aparece una normativa específica que regula el Sistema de Extensión (Ordenanza 388/11). La misma apunta al fortalecimiento y la jerarquización de la extensión, la cual entiende como el conjunto de acciones que pretenden articular el trabajo académico con el Estado y la comunidad para contribuir al desarrollo sustentable y de calidad de la sociedad. Asimismo, se propone integrar la extensión con la docencia y la investigación. La Ordenanza establece como agentes ejecutores de la extensión a los equipos de cátedras, departamentos, áreas, laboratorios, entre otros, dentro de la comunidad académica y, también, a personas u organizaciones ajenas a la Universidad, como ONG, empresas y otros colectivos u organizaciones. Ese detalle podría considerarse un avance con relación al interés institucional por promover la participación social. Y profundizando aún más en esa dirección, entre los criterios de evaluación de los proyectos se establece como relevante la incorporación de los destinatarios como coautores y participes de las distintas etapas de las propuestas — diagnóstico, diseño, ejecución y evaluación.

Como forma de fortalecer el trabajo en la temática, la UNER integra la REXUNI, cuyos objetivos son la coordinación de la extensión en el sistema universitario argentino con vistas a fortalecer las iniciativas, articular acciones, crear espacios de análisis y formación en la temática, revalorizarla e incluirla en el currículo universitario y los concursos docentes. A nivel regional, integra la AUGM, que cuenta con una Comisión Permanente de Extensión Universitaria desde 2012. Los objetivos de esta Comisión son: poner en debate la extensión como función sustantiva de la universidad a fin de promover su reconocimiento académico; generar masa crítica a través de la realización de capacitaciones, congresos y publicaciones sobre la temática; propiciar su impulso desde las políticas públicas e institucionales; identificar una agenda de problemáticas para el trabajo mancomunado en la región. 
En cuanto a su estructura, el Sistema de Proyectos de Extensión de la UNER establece diversas modalidades con diferentes características y objetivos: ${ }^{4}$

1) Programas de Extensión: propuestas de largo plazo que abordan aspectos relacionados con las problemáticas más urgentes de la sociedad. Tienen una duración de tres años pero, como están orientadas a asentar líneas de acción permanente en una determinada temática, en la actualidad se han prorrogado a tres años más.

2) Proyectos de Extensión: iniciativas de distinta índole — cultural, social, educativa y/o productiva - que aporten a la solución de los problemas sociales con objetivos a mediano y corto plazo. Es la línea más antigua y tradicional dentro del sistema; su duración es de un año.

3) Proyectos de Curricularización de la Extensión: experiencias orientadas a superar las condiciones de asimetría respecto de la docencia e investigación que plantean un trabajo que incorpore a la extensión como instancia curricular incluida en los planes de estudios de las facultades y cátedras. Se basan fuertemente en la formación de estudiantes y docentes.

4) Proyectos de Integración de las Funciones Universitarias "Prácticas Integrales y Territorio": se trata de una línea novedosa, derivada de la anterior, que apunta al desarrollo de prácticas donde converjan la extensión, investigación y docencia; promueven acciones en territorio y la interacción de docentes, estudiantes y actores sociales.

5) Proyectos de Extensión dirigidos por alumnos avanzados: están a cargo de estudiantes avanzados - con $70 \%$ de las materias aprobadas-, quienes son los responsables integrales bajo la supervisión de un codirector -docente que obra a modo de tutor-. Esta modalidad debe plantearse como acciones "extramuros" $\mathrm{y}$, preferentemente, participar a la comunidad en el diseño del proyecto.

6) Actividades culturales: suponen una acción inmediata a desarrollar en un cuatrimestre lectivo. Pueden estar a cargo de docentes o de los Centros de Estudiantes de las respectivas Unidades Académicas. Principalmente, configuran intervenciones de índole cultural y artística.

\section{Metodología de la investigación}

Al inicio del artículo se señaló que la escasez de investigaciones que toman a la extensión como su objeto de estudio, más allá de las sistematizaciones de experiencias y/o de la discusión de resultados de ciertas iniciativas, es un elemento inquietante para el desarrollo y fortalecimiento de las prácticas en el campo. En sintonía con ese planteamiento, el propósito de la tesis doctoral ya referida es llevar adelante un estudio empírico que, partiendo del análisis de las estrategias y acciones desplegadas en la UNER, constituya un aporte para su consolidación, profundización y reformulación. A continuación se describen los principales aspectos concernientes al diseño metodológico de la investigación, cuyos resultados preliminares se presentan en la próxima sección.

4) La descripción del Sistema es desarrollo propio del autor sobre la base de la Ordenanza 388/11 y los documentos de las convocatorias de las distintas modalidades, disponibles en el portal de Extensión de la UNER: https://extension.uner.edu.ar/ 
En términos generales, el estudio se plantea desde una perspectiva cualitativa; es de naturaleza exploratorio-descriptiva y evaluativa; tiene carácter sincrónico y transversal —abarca a las áreas centrales de la UNER y a todas sus Unidades Académicas-y está basado en fuentes primarias y secundarias.

El objetivo principal es generar un marco conceptual y metodológico para el análisis y potenciamiento de las prácticas de "apropiación social del conocimiento científico" que articule aportes de los estudios de la comunicación pública de la ciencia, la comunicación comunitaria y la tradición teórica y empírica de la extensión universitaria.

El abordaje empírico propiamente dicho, como se mencionó, contempla la recolección y análisis de información proveniente de informantes clave, documentos organizativos y de los proyectos de extensión, en dos niveles: a) un nivel macro, institucional; y b) un nivel micro, el de una selección intencional de proyectos.

a) En lo que concierne al nivel institucional, se examinaron tanto las normativas como los documentos vinculados directa o indirectamente con las políticas de extensión de la UNER. ${ }^{5}$ Asimismo, se están llevando adelante entrevistas en profundidad con las autoridades rectorales y de las Unidades Académicas responsables del área durante el período 2014-2018 con el fin de determinar, entre otras cuestiones, las estrategias políticas y los lineamientos con los cuales la Universidad encara el despliegue de la función; cuáles son los mecanismos de evaluación, promoción y apoyo que se ponen en juego; y de qué manera se vinculan y evalúan las experiencias y aprendizajes obtenidos de ellas con vistas a su retroalimentación.

b) Por lo que respecta al plano concreto de los programas, proyectos y acciones, a partir del Sistema de Proyectos de Extensión se conformó una muestra estratégica de experiencias iniciadas y finalizadas en el período comprendido entre los años 2013 y 2017. El recorte temporal obedece a la necesidad de contar con información completa relativa al desarrollo y resultados de las mismas, como también a la posibilidad de contrastar las valoraciones y conclusiones ex post del equipo responsable. De un total de 54 iniciativas desarrolladas en ese lapso, se seleccionaron intencionalmente 19 casos en función de un doble criterio de representatividad: por facultades, carreras y disciplinas; por la modalidad de la actividad según la clasificación institucional vertida en el apartado anterior. ${ }^{6}$ La muestra quedó conformada de la siguiente manera (ver Cuadro 1):

5) Resolución CS UNER 113/2005 (Estatuto UNER); Acuerdo Plenario CIN 650/2007 (Estatuto del Consejo Interuniversitario Nacional); Acuerdo Plenario CIN 681/2008 (Creación de REXUNI); Ordenanza CS UNER 388/2011 (Disposiciones generales del Sistema de Extensión UNER); UNER - Documento Preliminar de Discusión sobre la Curricularización de la Extensión; Resolución CS UNER 150 (Pautas Banco Interno de Evaluadores); Ordenanza CS UNER 411 (Régimen Becas de Extensión).

6) Las actividades culturales de facultades y centros de estudiantes no forman parte de los casos seleccionados ya que colocan su atención en iniciativas artístico-culturales y se alejan de las problemáticas ligadas a la apropiación de conocimientos científicos, aspecto central de la investigación. 
Cuadro 1. Corpus de la investigación.

\begin{tabular}{|c|c|c|}
\hline $\begin{array}{l}\text { Tipo/Período } \\
\text { de ejecución }\end{array}$ & Título & UU. AA. \\
\hline \multirow{3}{*}{$\begin{array}{l}\text { Proyectos de } \\
\text { curricularización } \\
(2015 \text { a 2016) }\end{array}$} & Prácticas Integrales de Cooperativismo. & $\begin{array}{l}\text { Facultad de Ciencias de la } \\
\text { Administración. }\end{array}$ \\
\hline & Compartiendo saberes y sabores en la escuela. & Facultad de Bromatología. \\
\hline & $\begin{array}{l}\text { Gestión de la tecnología médica en la red de atención de salud de la } \\
\text { ciudad de Diamante, Entre Ríos. }\end{array}$ & Facultad de Ingeniería. \\
\hline \multirow[t]{3}{*}{$\begin{array}{l}\text { Programas de Extensión } \\
\text { (2013 a 2016) }\end{array}$} & Jardín Botánico Oro Verde. & $\begin{array}{l}\text { Facultad de Ciencias } \\
\text { Agropecuarias. }\end{array}$ \\
\hline & $\begin{array}{l}\text { Participacion, Asociativismo y Educación Permanente con los adultos } \\
\text { mayores. }\end{array}$ & $\begin{array}{l}\text { Facultad de Ciencias de la } \\
\text { Educación. }\end{array}$ \\
\hline & Puerto Ciencia - Museo Interactivo de Ciencias. & Facultad de Ingeniería. \\
\hline \multirow{5}{*}{$\begin{array}{l}\text { Proyectos de Integra- } \\
\text { ción de las Funciones } \\
\text { Universitarias "Prácticas } \\
\text { Integrales y Territorio" } \\
\text { (2016 a 2017) }\end{array}$} & $\begin{array}{l}\text { Aprendizaje situado e integración de conocimientos a través de } \\
\text { experiencias en escuelas agrotécnicas de la provincia de Entre Ríos. }\end{array}$ & $\begin{array}{l}\text { Facultad de Ciencias } \\
\text { Agropecuarias. }\end{array}$ \\
\hline & $\begin{array}{l}\text { Formulación optimizada de premezclas para panificados libres de gluten, } \\
\text { de bajo costo, partiendo de la experiencia culinaria del taller de cocina } \\
\text { para celíacos de Acela, Entre Ríos. }\end{array}$ & Facultad de Bromatología. \\
\hline & $\begin{array}{l}\text { Periodismo en la universidad y las escuelas secundarias: las prácticas de } \\
\text { extensión, reflexiones en torno a los procesos de apropiación de conoci- } \\
\text { mientos en los estudiantes de la Orientación en Periodismo de Comuni- } \\
\text { cación Social y de secundaria. }\end{array}$ & $\begin{array}{l}\text { Facultad de Ciencias de la } \\
\text { Educación. }\end{array}$ \\
\hline & $\begin{array}{l}\text { La interacción del deterioro ambiental, la pobreza y las enfermedades. } \\
\text { Una alternativa participativa para su abordaje. }\end{array}$ & Facultad de Ciencias de la Salud. \\
\hline & Cárcel, Universidad y Sociedad. & Facultad de Trabajo Social. \\
\hline \multirow[t]{5}{*}{$\begin{array}{l}\text { Proyectos de Extensión } \\
\text { (2016 a 2017) }\end{array}$} & $\begin{array}{l}\text { Hacia una transformación en la organización productiva en materia de } \\
\text { seguridad alimentaria en la Región de Salto Grande. }\end{array}$ & $\begin{array}{l}\text { Facultad de Ciencias de la } \\
\text { Alimentación. }\end{array}$ \\
\hline & Fortalecimiento del diseño en emprendedores de base social. & $\begin{array}{l}\text { Facultad de Ciencias } \\
\text { Económicas. }\end{array}$ \\
\hline & Talleres de Comunicación Comunitaria con perspectiva de género. & $\begin{array}{l}\text { Facultad de Ciencias de la } \\
\text { Educación. }\end{array}$ \\
\hline & Prevención y detección temprana del cáncer colorrectal. & Facultad de Ciencias de la Salud. \\
\hline & $\begin{array}{l}\text { Espacios "con-sentidos". Abordaje y sensibilización en situaciones de } \\
\text { interpretación de Lengua de Señas Argentina Español en instituciones de } \\
\text { interés general y comunitario (2016). }\end{array}$ & Facultad de Trabajo Social. \\
\hline \multirow{3}{*}{$\begin{array}{l}\text { Proyectos de Extensión } \\
\text { dirigidos por alumnos } \\
\text { avanzados } \\
(2016 \text { a } 2017)\end{array}$} & Talleres de microemprendimiento en el barrio Antártida Argentina. & Facultad de Ciencias Económicas. \\
\hline & MOTI1 (2016). & Facultad de Ingeniería. \\
\hline & $\begin{array}{l}\text { "Mi gorra no es delito, mi piel no me define". Las juventudes y la } \\
\text { violencia institucional en el barrio Capibá de la ciudad de Paraná. }\end{array}$ & Facultad de Trabajo Social. \\
\hline
\end{tabular}

Fuente: elaboración propia. 
Para cada una de las iniciativas muestreadas se operó también en un doble plano: se analizaron sus respectivos registros documentales — proyecto, informe/s de avance e informe final- y se encuentran en desarrollo las instancias de recolección de información con los agentes participantes: entrevistas en profundidad con directores $y / o$ miembros del equipo de trabajo y grupos focales de discusión con los grupos o comunidades destinatarias. ${ }^{7}$

\section{Resultados preliminares}

Aquí se presentan los avances logrados hasta el momento producto del análisis documental -relativo a los proyectos (b) - y las entrevistas con los secretarios/as de extensión de las facultades y directores/as de las propuestas. Aunque parciales, el interés de los mismos radica en que ejemplifican de qué manera entendemos los posibles aportes a la mirada extensionista de una investigación que la toma como su objeto de estudio. Los resultados se presentan a partir de tres categorías. La primera trata de las concepciones sobre la extensión: ¿cuál o cuáles son las nociones de extensión presentes en los documentos y en el discurso de los entrevistados? ¿Con qué concepción y/o modelos expuestos se pueden relacionar? ¿Cuáles son los principales rasgos o características? La segunda refiere al surgimiento y antecedentes de las iniciativas. Indaga el recorrido de los proyectos: ¿de dónde surge? ¿Es una demanda de la comunidad, propuesta del equipo extensionista, o hay interacción entre los actores académicos y legos? La tercera analiza la interacción equipo/destinatario y otros participantes. Se observa la articulación que se propone entre los distintos actores de las iniciativas. Se presta especial atención al lugar que ocupan o que se espera de cada uno de los agentes: ¿quiénes participan de la instancia de armado y ejecución de las propuestas? ¿Cuáles son las intervenciones de los destinatarios en las propuestas?

Naturalmente, el primer aspecto a abordar en las entrevistas con los informantes giró en torno a su concepción de la extensión. Al respecto, al indagar entre los/as secretarios/as se encontró —en algunos casos explícita, en otros tácitamente- la visión como función sustantiva junto con la docencia e investigación como los tres pilares del accionar universitario:

"La extensión es una herramienta — que es una de las tres funciones principales de la universidad-". $(\mathrm{S} 1)^{8}$

Asimismo, todos los funcionarios coinciden en identificarla como la articulación de la universidad con la sociedad, posicionándose en general de manera crítica ante las experiencias unidireccionales de la academia a favor de instancias dialógicas-participativas. Así queda de manifiesto en algunos testimonios:

7) Aunque no se incluyen entre los resultados expuestos en este caso, la incorporación de la perspectiva de los destinatarios es un aspecto clave de la investigación propuesta, orientada precisamente a identificar procesos de "apropiación del conocimiento" más allá de los análisis estrictamente centrados en la oferta que se realiza desde las instituciones.

8) En los siguientes apartados se trabaja con los documentos de análisis y entrevistas realizados para la investigación. Con vistas a preservar su identidad, los entrevistados serán citados como S - haciendo referencia a los secretarios/as - D - para los testimonios de los directores/as de las iniciativas de extensión- y P — para citar pasajes textuales de los Proyectos-. Los números corresponden a las personas entrevistadas y documentos analizados que se mantienen anónimos. 
"La universidad tiene que dar respuestas concretas a los problemas reales de la comunidad (...). Para resolverla no podemos hacer un abordaje de tipo paternalista [sino] trabajar con la comunidad. O sea, detectar en conjunto con la comunidad los problemas y aportar desde la universidad todos los recursos - humanos y materiales- para tratar de resolver el problema, pero con un rol protagónico de la comunidad". (S2)

Algunos mencionan explícitamente la extensión crítica como modelo a seguir, el cual propicia un encuentro dialógico entre los actores que producen colectivamente:

"Adhiero al paradigma de la extensión crítica (...). Esa experiencia de estar en situación y en coproducción es lo que te hace pensar, crecer, pensar soluciones, tejer redes para trabajar con otro". (S3)

Actualmente, los secretarios se encuentran discutiendo una nueva ordenanza sobre extensión que reemplace la vigente. En su reflexión sobre los desafíos actuales de la extensión, los entrevistados refieren a la jerarquización, curricularización e integración como objetivos que, si bien se fomentan desde las políticas de la Universidad, es necesario reflejar en un nuevo marco normativo:

"Estamos reviendo algunas cuestiones en la ordenanza (...). El sistema lo que tiene que hacer es favorecer que la problemática ingrese al campo universitario". (S4)

Otra de las razones esgrimidas sobre la necesidad de generar una nueva ordenanza se debe a que en la actual no están incorporadas las líneas de proyectos de Curricularización, Integralidad y Alumnos Avanzados — que se fueron generándose de 2012 en adelante-, lo que queda claro a partir de la reflexión de uno de los entrevistados:

"No solo va a reflejar lo que se ha innovado en extensión sino, también, va a quedar planteada cuál es la normativa que la UNER promueve cuando habla de extensión". (S5)

A nivel de las experiencias analizadas, el primer dato que demanda atención es la escasa, y en algunos casos nula, mención del concepto de extensión en los documentos de los proyectos. A lo largo de los textos no se observa referencia alguna. En ocasiones aparece solo de manera nominal para referirse a la propuesta o línea de financiamiento en la cual se enmarca. En otros, se desarrolla de manera escueta como vínculo que la Universidad mantiene con la comunidad, orientándose a una visión paternalista/transferencista. Finalmente, hay casos en los que se explicitan vagamente otros conceptos, como responsabilidad o compromiso social:

"Mostrar el papel que juega la Universidad como promotora (...) a través de diversas estrategias en el marco de la Responsabilidad Social Universitaria". (P1)

Esta situación no se repite en las entrevistas a los directores, quienes reconocen sus propuestas como extensionistas y pueden desarrollar una conceptualización de la misma. Sin embargo, en algunos casos las respuestas son breves y evidencian cierta falencia en la reflexividad en el campo. Por ejemplo:

"Viene la gente a que nosotros podamos mostrarles lo que nos interesa (...). Por eso nosotros hablamos de extender el conocimiento (...). Estamos difundiendo, enseñando, formando. Eso es lo que hacemos". (D1) 
En los casos en que efectivamente se profundiza en una conceptualización de la extensión, se puede vislumbrar de qué manera los distintos modelos, características y estrategias identificadas en la construcción teórica operan conjuntamente. En general, se entiende como articulación de la Universidad con la sociedad, en ocasiones, desde un punto de vista dialógico y bajo la concepción de ecología de saberes, acercándose a la idea de la tradición reformista:

"El diálogo de los ámbitos que integran la Universidad con la comunidad. Poner en juego

los saberes que la Universidad genera con los saberes de la comunidad". (D2)

Por otro lado, se comprenden, desde un punto de vista difusionista, las experiencias como transmisión de saberes académicos a la comunidad:

"Es llevar a la práctica y a la sociedad lo que uno aprende en la facultad y volcarlo de alguna manera". (D3)

En todos los casos está presente la idea de que la Universidad aporta sus conocimientos para solucionar distintas problemáticas de las comunidades. La ciencia no se pone en cuestión, más bien sus conocimientos son considerados como aspectos que contribuyen al desarrollo social:

"Mediante el contacto fluido con los actores relevantes de cada una de las organizaciones y de diferentes organizaciones entre sí, se espera que se generen propuestas de resolución de situaciones problemáticas o de oportunidades de mejoras en las mismas". (P1)

Se hace énfasis en la extensión como experiencia formativa del estudiante, a partir de la cual este obtiene experiencias sobre las realidades sociales que complementan la enseñanza profesional. Esto supone, asimismo, que el alumno que participa en la extensión genera un plus respecto de sus compañeros que solamente se centran en cumplir las responsabilidades curriculares de la carrera académica:

"Intentamos construir una nueva forma de hacer extensión universitaria, donde el alumno como actor protagónico, pueda alcanzar escenarios complejos de anclajes discursivos y prácticos que configuran la conciencia individual y colectiva en el contexto en el cual él se encuentra inmerso como individuo y como miembro de la institución". (P2)

Por su parte, los estudiantes que están a cargo de los proyectos sostienen esta comprensión de la extensión como aprendizaje y complemento de su carrera:

"Es una iniciativa innovadora y sumamente enriquecedora como espacio de aprendizaje". (P3)

También hay docentes que destacan el aporte de las experiencias como elemento que contribuye a su formación y las tareas que se desarrollan en clases, y colocan el énfasis en la posibilidad que otorga la extensión de poner en práctica los conceptos teóricos:

"Uno puede estar estudiando pero tiene que preguntarse qué de eso es aplicable a la realidad y cómo lo transformás para aplicarlo a la realidad. ¡Creo que la extensión es la pata fuerte!". (D4) 
"La extensión es una forma de aprender para los profesores y estudiantes universitarios. Es realmente un espacio de aprendizaje." (D5)

Los entrevistados reconocen la falta de sistematización de estudios sobre extensión y la urgencia de desarrollar investigaciones sobre la temática. En muchos casos se expresa la necesidad de generar un conocimiento metódico y sistemático:

"La devolución de la experiencia de trabajar en extensión es una producción de conocimiento totalmente interesante (...). Si uno pudiera tomárselo más enserio y volcarlo en una sistematización sería aporte importante al conocimiento". (D6)

En cuanto a la integralidad de funciones, se menciona a modo de estrategia pero no se describe con profundidad ni se plantea concretamente:

"Se espera asimismo que de la experiencia (...) surjan posibles problemáticas a ser abordadas por investigaciones futuras aplicadas al sector, con la finalidad de dar respuesta a demandas específicas del medio. Asimismo se intenta dar respuesta a necesidades de formación existente en nuestra provincia [sobre la temática] mediante el desarrollo de cursos o programas de posgrado". (P4)

Sobre el surgimiento de los proyectos, en todos los casos son propuestas que parten desde la academia, son planificadas por la comunidad universitaria y ejecutadas por esta. En muchos de esos casos existe un trabajo previo a la propuesta a partir del cual se recogen los reclamos y necesidades de las comunidades para definir la problemática a abordar, pero la comunidad no interviene en su elaboración o gestión. Sin embargo, se puede distinguir entre aquellas que son preocupaciones desde el sector universitario e iniciativas que intentan dar respuestas a demandas de la comunidad que implican un mayor grado de apertura hacia el trabajo conjunto con la sociedad. Encontramos entre los documentos:

“(...) lograr la accesibilidad de toda la población (...) al programa y garantizar la calidad de las intervenciones y los procesos, con un monitoreo y evaluación permanentes e impartir la enseñanza para la formación de nuevos y mejores profesionales, buscando conocimientos nuevos para poder transferir los mismos a la sociedad y que pueda servir para el mejoramiento de la calidad de vida". (P5)

Mientras que, en otras ocasiones, se señala como demanda latente en la comunidad:

“(...) mujeres de diversos barrios [manifestaron] la necesidad inmediatas de seguir avanzando sobre estas temáticas. A partir de esta necesidad (...) nos acercamos al barrio para acercar nuestra propuesta de talleres, donde nos recibieron con gran entusiasmo y expectativas de realizar un trabajo conjunto". (P6).

Algunos testimonios evidencian una doble incidencia de las propuestas, originadas tanto desde la Universidad como de la sociedad, lo que explican como un trabajo de escucha y aprendizaje a partir de las experiencias:

"Es parte y parte. Honestamente, hay mucha de estas cosas que fueron originariamente iniciativas de los integrantes [universitarios] (...). Con el tiempo, la experiencia y el contactos que hemos tenido (...) nos ha permitido ir relevando algunas necesidades". (D7) 
"En algún momento cometimos el error de la pretensión de ir con la solución. Sin embargo, en la experiencia fuimos tomando lo que decía cada una de las partes para ver en qué podíamos aportar." (D8)

En lo que concierne al rol de las comunidades destinatarias, se puede observar el protagonismo que los funcionarios dan a las mismas. En primer lugar, algunos muestran desacuerdo con la propia denominación ya que indica un rol pasivo de los agentes:

"Si hablás de destinatarios ya lo estás poniendo afuera. Seguimos reproduciendo aquella lógica de que la universidad es el que sabe y la que tiene (...). Es una cosa que cuestiono históricamente de toditos los formularios de los proyectos". (S7)

Se trata de generar un trabajo conjunto donde los actores sociales tengan una participación activa en la resolución de las distintas problemáticas:

"Me parece muy bien que en los proyectos de extensión figuren como integrantes, teniendo un rol protagónico, personas externas a la Universidad y que pertenezcan a las instituciones con las que la Universidad se vincula a través de los proyectos". (S8)

"No solo salir nosotros con nuestras agendas sino empezar a construir las agendas de propuestas en función de las necesidades del afuera." (S9)

No obstante, tal como se infiere del siguiente fragmento, entienden que la participación e inclusión de los distintos actores comunitarios aún no está resuelta y, en la práctica, se continúa con una idea verticalista de extensión:

"Todavía nos falta muchísimo trabajar la demanda, muchas veces (...) seguimos trabajando con la oferta". (S3)

Esto puede atribuirse a diferentes razones pero, en principio, se reconoce una dificultad teórico-práctica de incorporación genuina de las comunidades:

"Lo que todavía nos cuesta es la mirada del otro. Está bastante sesgada porque lo analizamos con nuestras categorías y formación. Nos cuesta entender el rol del otro (...). Si bien se trabaja con el otro jla producción la hago yo!". (S3)

Finalmente, existe una diferencia sustantiva entre los discursos de los directores, en los cuales gran parte de ellos asegura realizar una labor horizontal e inclusiva de los destinatarios, y la organización de las actividades que plantean en la confección de los documentos. En cuanto a las funciones de cada actor, los proyectos distinguen entre: aquellos que son ejecutores de los proyectos -universitarios; las organizaciones participantes que aportan recursos para el desarrollo de las acciones-; y los destinatarios - la comunidad específica a la cual se dirigen las propuestas-. En tanto, desde el punto de vista de las dinámicas y metodologías de las actividades que se proponen, la mayoría posee lógicas paternalistas y verticalistas, desde la Universidad a la sociedad. Por un lado, se realizan propuestas de capacitación en diferentes temáticas y, por otro, se organizan instancias de diagnóstico e intervención profesional. Además, hay iniciativas de muestras y/o excursiones guiadas. En todos los casos, la participación de la comunidad se da en cuanto a proponer la temática 
a abordar —o bien esta es recogida por el sector académico como trabajo de escucha de las necesidades sociales- y a partir de la apertura de las actividades que, en ocasiones, se piensan como participativas e interactivas.

Ahora bien, en las entrevistas y en algunos pasajes de los documentos se encuentra una clara predisposición al trabajo en conjunto con la sociedad:

"Es una falacia la visión de la universidad como proveedora de saber. ¡No es así! Hay una cuestión de construcción conjunta de intereses, inquietudes, problemas". (D9)

"Nos propusimos llevar adelante este proyecto en conjunto con los jóvenes del barrio." (P7)

Algunos, incluso, mencionan el diálogo entre saberes:

"Coordinar acciones entre diversas instituciones en pro de potenciar fortalezas (...) a través del intercambio cultural y saberes populares de las comunidades involucradas para lograr transformar la organización productiva". (P8)

"Poner en juego los saberes que la Universidad genera con los saberes de la comunidad." (D10)

\section{Conclusiones}

Puede observarse, a partir de los antecedentes mencionados, un cuerpo de estudios que abordan la extensión latinoamericana como campo problemático particular. A partir de estos se comprende la extensión como la articulación de la universidad con la sociedad, que en su devenir histórico tomó los desafíos de la academia tendientes a generar una mayor apertura hacia las comunidades de la cual forma parte y la sustenta. Se distinguen como antecedentes de gran relevancia los movimientos estudiantiles generados luego de la experiencia de 1918 y, en la actualidad, se propone llevar adelante un segundo proceso reformista que profundice las propuestas pioneras e introduzca los desafíos coyunturales de las sociedades del capitalismo global e informacional. Con vistas a generar una democratización del conocimiento científico y las universidades, se debe gestar un proceso en donde se jerarquice la extensión y se coloque junto con las demandas sociales —en particular de aquellos sectores vulnerables.

Las propuestas del campo tienen su correlato en las políticas universitarias de la región que, en muchos casos, buscan el fortalecimiento de la extensión. El caso de la UNER demuestra esta consonancia con los aportes teóricos puesto que desde sus políticas y normativas trabaja en la jerarquización, curricularización e integración de funciones, haciendo énfasis en el trabajo en red con otras instituciones nacionales e internacionales. Asimismo, interpela un trabajo inclusivo y horizontal con la sociedad. Por su parte, los actores de la Universidad acompañan estos objetivos que, sin embargo, parecen estar más resueltos a nivel discursivo y no tanto en las prácticas que estos generan. Podemos comprender que la posición que toman los entrevistados está intrínsecamente relacionada con las teorías en el campo pero ello no sucede de la misma manera en cuanto al armado y ejecución de las propuestas, donde prevalecen tendencias de tipo paternalistas, asistencialistas y verticalistas. 
No obstante, cabe destacar la coexistencia de acciones participativas e inclusivas de mayor apertura hacia las comunidades.

Hasta el momento, la investigación aporta al campo de la extensión latinoamericana al desarrollar una conceptualización del mismo a partir de las principales tendencias y características que tiene en la región. También abona a la comprensión de los planteamientos actuales en la problemática desde un caso particular, como la UNER y, por último, el análisis de los actores que intervienen en los procesos ayuda a entender cuáles son los sentidos generados sobre la extensión, en qué consisten las propuestas y cómo se produce la articulación universidad-sociedad. Resta completar la etapa de análisis documental y entrevistas, a partir de lo cual se podrán establecer con mayor precisión las posturas de los diferentes actores universitarios, las estrategias que llevan a cabo y la incidencia del campo disciplinar de procedencia en las mismas. En tanto, los grupos focales con los destinatarios de las propuestas aportarán a conocer cómo vivencian las experiencias de extensión las comunidades no universitarias, qué piensan de las mismas, si se sienten interpeladas a participar y si se apropian o no de los conocimientos científicos.

Como se señaló en la Introducción, pese al enorme cuerpo de reflexiones sobre la temática y las políticas que lo acompañan, actualmente hay una carencia de estudios que aborden la extensión como su objeto. Es por ello que este artículo presentó una propuesta como forma de identificar las contribuciones que pueden hacerse al campo desde la investigación. La importancia de la generación de conocimientos en este rumbo es complementaria a los objetivos que se establecen para las universidades y la extensión en las sociedades actuales. Para finalizar, se propone una serie de interrogantes susceptibles de ser abordados desde una agenda de investigación a nivel local, nacional y regional, tales como: las experiencias y desarrollo histórico de las políticas, conceptos y prácticas sobre extensión; la actualidad de las experiencias extensionistas: ¿qué temáticas o problemas abordan? ¿Quiénes son sus actores? ¿Qué aportes o producciones pueden identificarse de las mismas? ¿Cuáles y cómo son las políticas universitarias en el campo y cómo se articulan en las prácticas? Las evaluaciones de los proyectos: ¿qué evaluaciones se hacen? ¿Son coercitivas o abiertas? La extensión como formación y antecedente universitario: ¿cuáles son los aprendizajes y aportes a la formación puestos en juegos en la extensión? ¿Cómo se visibiliza la extensión en el currículo de las universidades y los concursos docentes? ¿Cómo se puede generar una integración de las funciones que vincule las distintas instancias del trabajo universitario con articulación y simetría? Todos estos interrogantes, entre otros, contribuirían al campo de la extensión latinoamericana.

\section{Referencias bibliográficas}

Arocena, R. (s/f). Curricularización de la extensión: ¿por qué?, ¿cuál?, ¿cómo? En: Integralidad, tensiones y perspectivas. Cuadernos de Extensión No 1. Comisión Sectorial de Extensión y Actividades en el Medio. Recuperado de: http://www.extension.udelar.edu.uy/wp-content/uploads/2017/11/Cuaderno-n\%C2\%B01-integralidad.pdf (11/06/2018).

Beltramino, T. y Theiler, J. (2017). Extensión universitaria e innovación social: reflexiones en torno a los vínculos entre la universidad y los actores sociales. Revista +E versión en línea, 7(7), 84-96. Santa Fe, Argentina: Ediciones UNL. 
Bolaños, S.; Lara Campos, C. Villalobos Araya, A. (2017). Desarrollo de la extensión en las universidades estatales costarricenses. Influencias, modelos y desafíos actuales. Revista +E versión en línea, 7(7), 122-131. Santa Fe, Argentina: Ediciones UNL.

Cano Menoni, J.A. (2014). La extensión universitaria en la transformación de la universidad latinoamericana del siglo XXI: disputas y desafíos. Buenos Aires: Clacso. Recuperado de: http://biblioteca.clacso.edu.ar/clacso/ becas/20141202093928/ensayo_cano_premio_pedro_krotsch.pdf (11/06/2018).

Castro, J.O. y Tommasino, H. (Comps.) (2017). Los caminos de la extensión en América Latina y el Caribe. Santa Rosa: Universidad Nacional de La Pampa. Recuperado de: http://www.unlpam.edu.ar/cultura-y-extension/edunlpam/catalogo/publicaciones-de-extension-universitaria/los-caminos-de-la-extensi\%C3\%B3n-en-am\%C3\%A9rica-latina-y-el-caribe (11/06/2018).

Castro, J.O. y Oyarbide F. (Comps.) (2015). Los caminos de la extensión en la universidad argentina. Santa Rosa: Universidad Nacional de La Pampa. Recuperado de: http://www.unlpam.edu.ar/images/extension/Los\%20 caminos\%20de\%20la\%20extension.pdf (11/06/2018).

Cecchi, N.H., Pérez, D.A., Sanllorenti, P. (2013). Compromiso social universitario. De la Universidad posible a la Universidad necesaria. CONADU-Instituto De Estudios y Capacitación (IEC). Recuperado de: http://conadu. org.ar (11/06/2018).

Cortassa, C. (2012). La ciencia ante el público. Dimensiones epistémicas y culturales de la comprensión pública de la ciencia. Buenos Aires: Eudeba.

Freire, P. (1970). Pedagogía del Oprimido. México: Siglo XXI Editores.

González López, B. (2017). Extensión universitaria en Chile: discursos y prácticas sobrevivientes. Revista $+E$ versión en línea, 7(7), 110-121. Santa Fe, Argentina: Ediciones UNL.

Lossio, O. y Ruben, A.B. (2017). Las voces de estudiantes del Profesorado de Geografía sobre la inclusión curricular de la extensión. Revista +E versión en línea, 7(7), 296-307. Santa Fe, Argentina: Ediciones UNL.

Menéndez, G. (2017). Resignificación de la extensión a 100 años de la Reforma Universitaria de 1918.

Revista $+E$ versión en línea, 7(7), 24-37. Santa Fe, Argentina: Ediciones UNL.

Pérez Lindo, A. (2003). Universidad, conocimiento y reconstrucción nacional. Buenos Aires: Biblos.

Sousa Santos, B. de (2007). La Universidad en el siglo XXI. Para una reforma democrática y emancipatoria de la universidad. Bolivia: Editores Plural. Recuperado de: http://www. boaventuradesousasantos.pt/media/universidad_siglo_xxi-.pdf (11/06/2018).

Tommasino, H. y Cano, A. (2016). Modelos de extensión universitaria en las universidades latinoamericanas en el siglo XXI: tendencias y controversias. Universidades, (67, enero-marzo), 7-24. Recuperado de: http://oai.redalyc.org/articulo.oa?id=37344015003 (11/06/2018).

Unión Latinoamericana de Extensión Universitaria (ULEU) (2015). Glosario de términos que se utilizan en extensión universitaria. Puyo, Ecuador: Universidad Estatal Amazónica. Recuperado de: http://www.uleu.org/ uleu_wp/2015/08/31/libros-recomendados/ (11/06/2018).

Tünnermann Bernheim, C. (2003). La universidad latinoamericana ante los retos del siglo XXI. México: Unión de Universidades de América Latina.

Valsagna, M.V. (2009). La Extensión Universitaria en los '60: debates y disputas en torno a la transformación social. El caso de la Universidad Nacional del Litoral. Una experiencia de comunicación, culturay desarrollo. Tesis de Licenciatura en Comunicación Social, Universidad Nacional de Entre Ríos. 